\title{
Claudia Kaluza
}

Pädagogische Hochschule Wien

\section{Bernhard Schimek}

Pädagogische Hochschule Wien

\section{Diversität und Schulentwicklung}

\section{in der Trias von Wertediskurs, Evidenzbasierung und Bildungstheorie}

DOI: https://doi.org/10.53349/sv.2021.i2.a86

\begin{abstract}
Gesellschaftliche Entwicklungen wie beispielsweise Globalisierung, Pluralisierung von Lebensformen (Walgenbach 2017, S. 7) wirken auf die Institution Schule und ihre Beteiligten. Diversität zeigt sich dabei nie neutral, sondern ist mit Bildungschancen und -risiken verbunden. Akteur*innen von Schule sind daher gefordert, diversitätsbezogene Herausforderungen proaktiv zu gestalten. Ausgleichsbestrebungen gibt es auf systemischer Ebene, z.B. durch die Zuweisung von Ressourcen. Manche Benachteiligungen bleiben jedoch unberührt oder unerkannt. Diskriminierung und Privilegierung sind im Umgang mit Diversität jene Pole, die sich im pädagogischen Alltag mehr oder weniger offensichtlich zeigen (Kaluza \& Schimek 2017, S. 11f). Schulen stehen im Rahmen von Schulentwicklung vor der Aufgabe, im Einklang mit den grundgesetzlichen Vorgaben, dem Qualitätsrahmen für Schulen und den systemischen Anforderungen, Ansprüchen im Kontext von Diversität gerecht zu werden.
\end{abstract}

Diversität, Schulentwicklung, Bildungsgerechtigkeit

\section{Einleitung}

Gesellschaftliche Entwicklungen wie Globalisierung, Migration, Europäische Integration, Pluralisierung von Lebensformen bzw. Lebensstilen, demographischer Wandel oder der gesellschaftliche Einfluss sozialer Bewegungen (Walgenbach 2017, S. 7) wirken sich auch auf die Institution Schule und somit auf alle an Schule beteiligten Akteur*innen aus. Diversität zeigt sich dabei nie neutral, sondern ist mit Bildungschancen und -risiken verbunden, wie auch internationale Schulleistungsuntersuchungen (bspw. PISA) thematisieren (bspw. OECD 2018, Suchań \& Itzlinger-Bruneforth 2017). Akteur*innen von Schule sind daher gefordert, Heraus- 
forderungen im Kontext von Diversität, proaktiv zu gestalten. Auf systemischer Ebene intendiert das Bildungssystem, z.B. durch Zuweisung von Ressourcen, einzelne Benachteiligungen auszugleichen. Andere bleiben hingegen unberührt, unerkannt oder unbeachtet. Diskriminierung und Privilegierung bzw. Anerkennung sind im Umgang mit Diversität jene Pole, die sich im pädagogischen Alltag mehr oder weniger offensichtlich zeigen (Kaluza \& Schimek 2017, S. 11f). Gesellschaftliche Macht-, Privilegierungs- und Diskriminierungsverhältnisse wirken auf die Institution Schule und ihre Entwicklung. Diversitätsbewusste, diskriminierungsfreie Schulentwicklung setzt daher die Auseinandersetzung mit Fragen der Bildungsgerechtigkeit voraus. Darüber hinaus stehen Schulen im Rahmen von Schulentwicklung u.a. vor der Aufgabe, im Einklang mit den grundgesetzlichen Vorgaben und systemischen Anforderungen, Ansprüchen im Rahmen von Diversität gerecht zu werden. Deutlich gemacht wird diese schulische Herausforderungen anhand des folgenden Beispiels, welches durchgehend aufgegriffen wird:

Die Schule $X$ wird alltagssprachlich als Brennpunktschule bezeichnet, da mehrheitlich Schüler*innen aus sozial benachteiligten Familien diese Schule besuchen. Gleichzeitig ist für Österreich bekannt, dass der Bildungserfolg stark mit der sozialen Herkunft verknüpft ist. Schule $X$ steht vor der Herausforderung, mit der Frage umzugehen, ob bzw. inwieweit Schüler*innen aus sozial benachteiligten Familien mit diskriminierenden Effekten konfrontiert sind.

\section{Bildungsgerechtigkeit und Systementwicklung}

OECD-Auswertungen der PISA-Studie 2009 beschreiben bereits 2011 einen positiven Zusammenhang zwischen dem Grad der Autonomie der Schulstandorte bei der Festlegung und Gestaltung der Unterrichtsinhalte (Kursangebote, Auswahl der Schulbücher, ...), der Beurteilungsmethoden und der Ressourcenallokation sowie den Schüler*innen-Leistungen. Insbesondere gilt dies in Verbindung mit Rechenschaftspflicht (OECD 2011, S. 113). Es ist daher davon auszugehen, dass nationale Bildungssysteme zusehends höhere Grade an Autonomie und Verantwortlichkeit realisieren werden (siehe z.B. Bildungsreform 2017 in Österreich). „[E]videnzbasierte Steuerung der standortbezogenen Schulentwicklung“ (BMBWF 2019, S. 36) realisiert - im Sinne des diversitätsorientierten Wirkungszieles „Verbesserung der Bedarfsorientierung der Bedarfsorientierung sowie der Chancen- und Geschlechtergerechtigkeit" (BMBWF 2019, S. 5) - die Möglichkeit, systematisch systemische Ungerechtigkeitsfaktoren zu identifizieren, wie z. B. der erschwerte Erwerb von Grundkompetenzen durch Schüler*innen aus sozial benachteiligten Verhältnissen, wenn entsprechende Datenerhebungen damit verbunden sind.

Konzepte von Bildungsgerechtigkeit suchen Antworten auf die Frage, bei wem unter welchen Voraussetzungen, mit welchen Zielen und Mitteln die ungleiche Verteilung von Bildungschancen ausgeglichen werden soll (Dederich 2016, S. 93). Bildungsungleichheiten sollen reduziert, Bildungserfolg und damit schulische Selektion und Allokation von askriptiven Merkmalen (Geschlecht, Ethnie, Bildung/Beruf/Einkommen der Eltern, ...) entkoppelt werden (Bruneforth et al. 2012, S. 189; Giesinger 2011, S. 422; Rawls 1975). 
Je stärker Schulen für ihre Entwicklung und ihren Erfolg verantwortlich gemacht werden, desto mehr Bedeutung gewinnt Schulentwicklung für den einzelnen Standort und desto mehr werden Fragen der Bildungsgerechtigkeit in die Verantwortung der Einzelschule übertragen. Die damit verbundene Komplexität lässt sich mit dem „School-Governance-Konzept“ auf drei Ebenen adressieren (Althoff 2008, S. 124).

Die Makroebene umfasst das staatlich und/oder regional gesteuerte Schulsystem, dessen "strategische Zielsetzungen“, „Verfahrensvorschriften, Lehrpläne und Ressourcenzuweisungen"sind . Durch diese werden Kompositionseffekte wirksam. Je höher die soziale Benachteiligung einer Schule, desto schlechter sind die Leistungen der Schüler*innen (Bruneforth et al. 2012, S. 202), insbesondere bei Ressourcenverteilung gemäß formaler Chancengleichheit, welche durch Gleichverteilung zur Schlechterstellung einzelner Schüler*innen bzw. -Gruppen führt. Schulen sehen sich auf der Makroebene demnach mit Effekten konfrontiert, deren Wirkungen nur in bedingtem Ausmaß auf der Mesoebene durch die einzelne Schule ausgeglichen werden können, welche u.a. die Gestaltung von Entwicklungsplänen (pädagogische Profilbildung) sowie die Gewährleistung deren Umsetzung inkl. Allokation der zur Verfügung stehenden Ressourcen sowie die Verantwortlichkeit gegenüber den erzielten Ergebnissen (Althoff 2008 , S. 124) umfasst. Institutionell handlungsleitend erscheint Rawls' Differenzprinzip, welches fordert "die Anstrengungen auf die Verbesserung der langfristigen Aussichten der am wenigsten Bevorzugten zu lenken“ (Rawls 1975, S. 122). Entsprechend einer „Forderung nach einer angemessenen Bildung für alle" (Giesinger 2011, S. 425) ist durch Schulentwicklung außerdem ein Bildungsminimum, also ein Mindestmaß, welches die Teilhabe am gesellschaftlichen, wirtschaftlichen, politischen Leben gewährleistet, sicherzustellen (Bruneforth et al. 2012, S. 189). Damit wird für diversitätsbewusste und diskriminierungsfreie Schulentwicklung die Gewährleistung der "Anerkennungsformen des Respekts und der Wertschätzung" (Stojanov 2011, S. 42) adressiert.

Mit steigendem Autonomiegrad von Schulen gewinnt für diversitätsbewusste Schulentwicklung die Auseinandersetzung mit dem Differenzdilemma stetig an Bedeutung. Je mehr die Organisation und Gestaltung des Unterrichts, insbesondere der Ressourcenallokation in den Verantwortungsbereich des einzelnen Schulstandortes übertragen wird, desto mehr kumuliert auf der Mesoebene die ethische Auseinandersetzung mit dem Differenzdilemma. Werden Differenzen über Kategorisierungen thematisiert, werden „Ausschlussprozesse entlang von Differenzkategorien“ (Dahmen \& Vollmer 2018. S. 85f) thematisierbar, institutionelle Diskriminierungen adressierbar und Handlungsoptionen konzipier-, argumentier- und priorisierbar. Es besteht dabei allerdings die Gefahr, dass Differenzen statisch aufgefasst, LabellingProzesse verfestigt und damit Privilegierungs- und Diskriminierungsverhältnisse bestätigt bzw. verstärkt werden.

Auf der Mikroebene bedeutet die Veränderung der Steuerungsmechanismen verstärkte Teamarbeit und eine Intensivierung der gemeinschaftlichen Verantwortlichkeit für den Systemoutput (Althoff 2008, S. 124). Insgesamt gilt es, die sozialen Inklusionsbedingungen innerhalb einer Schule mehrdimensional (Eintrittsmöglichkeiten in die Schule, tätigkeitsbezogen, Partizipationsmöglichkeiten innerhalb der Schule, horizontale/vertikale Inklusionschancen, ...) zu bestimmen und „das ,schlichte Köpfezählen“ bestimmter als diversitätsrelevanter 
Zielgruppen um Vorstellungen von Inklusionsqualität anzureichern" (Dobusch 2017, S. 6263). Ziel ist, dass allgemeine Qualitätsvorstellungen gegenüber Stakeholdern klar artikulierbar, Bildungsungerechtigkeiten analysier- und identifizierbar (z.B. geringerer Bildungserfolg aufgrund askriptiver Merkmale), eine zielgruppenspezifische schulische Fokussierung auf die „am wenigsten Bevorzugten“ (Rawls 1975, S. 122) argumentierbar und die Wirkungen gesetzter Maßnahmen messbar werden.

Auf der Makroebene hat Schule X die Verpflichtung, z.B. die strategische Zielsetzung des Qualitätsrahmens für Schulen eine "[...] gender- und diversitätsgerechte Schulkultur" und darüber hinaus "die damit verbundenen Werte und Haltungen" zu vertreten. Gleichzeitig führen Verfahrensvorschriften für die Aufnahme von Schüler*innen, das Einzugsgebiet der Schule, ... zu einer besonders herausfordernden Schüler*innen-Zusammensetzung an Schule X. Auch wenn Schule X dies kaum verändern kann, muss die Schule X auf der Mesoebene Überlegungen auf Basis von Schuldaten anstellen und Maßnahmen setzen, um Ungerechtigkeiten aufgrund von sozialer Herkunft zu vermeiden und dafür zu sorgen, dass alle Schüler*innen bestmöglich für ihre Teilnahme am gesellschaftlichen Leben vorbereitet werden. Dazu gehört, wie im Qualitätsrahmen für Schulen vorgesehen, besonders „den professionellen Umgang mit Vielfalt durch den Aufbau von [...] Diversitätskompetenz" zu fördern. Sowohl auf Meso- als auch auf Mikroebene bedeutet dies, als Schule darauf zu schauen, ob bzw. wo Schüler*innen aus sozial benachteiligten Familien ausgeschlossen werden (z.B. aufrund von Unterrichtsinhalten, der verwendeten Sprache durch die Pädagog*innen, Praktiken wie Schulaktivitäten oder das Selbstverständnis der Schule an sich). Als ersten Schritt vereinbaren die Schulleitung und alle Lehrer*innen, dass bei der Teilnahme an Lehrausgängen sichergestellt wird, dass niemand aufgrund finanzieller Probleme ausgeschlossen wird (sehr frühe Ankündigung eines Beitrages, niederschwelliges Angebot der Unterstützung durch Elternverein, ...).

\section{Basis diversitåtsbewusster Schulentwicklung}

Ein produktiver Umgang mit Diversität in der Schulentwicklung kann am einzelnen Schulstandort nur der gesamten Schule als Organisation gelingen. Es bedarf der Entwicklung von Strukturen, die zuerst an den Bedürfnissen des Subjekts ausgerichtet sind und die Partizipation aller an schulische beteiligten Akteur*innen ermöglichen (Saalfrank 2008, S. 145; Rolff 2006, S. 332; Althoff 2008, S. 128). Schulen müssen folglich dabei unterstützt werden, einen strukturierten Blick auf diskriminierende und anerkennende Effekte am eigenen Schulstandort richten zu können (Kaluza \& Schimek 2017, S. 11f). Um dahingehend schulische Evaluation, wie sie auch im Qualitätsrahmen für Schulen vorgesehen ist (BMBWF o.J.), sicherzustellen, sind entlang bestehender Konzepte und Instrumente schulstandortbezogen und systematisiert (unter Einbezug von Sichtweisen aller Akteur*innen) weitere diversitätsspezifische Fokussierungen einzunehmen (Demski 2017, S. 38f), um der Gefahr einer Engführung gezielt entgegenzutreten. Systemische Normierungen müssen besonders hinsichtlich Leerstellen nicht im Fokus stehende, aber dennoch im Kontext des Bildungssystems maßgeblich Relevanz 
entfaltenden Diversitätsaspekte (z. B. Armutseffekte, das Auftreten von genderbezogenem oder trans- und homophobem Bullying, ...) - befragt werden.

Angestrebt wird mit Evidenzbasierung im Kontext von Schulentwicklung eine Objektivierung der pädagogischen Arbeit, die auch zu einer Abkehr von einzelnen Akteur*innen von einem Handeln auf Grundlage von Erfahrungswissen erfordern kann (Demski 2017, S. 38f). Maßgebliches Ziel ist die Stärkung der pädagogischen Handlungskompetenz durch handlungsleitende, objektivierte Informationsquellen entlang bildungstheoretischer Rahmungen, wobei die unterschiedlichen Ebenen von Bildungsgerechtigkeit jeweils Beachtung finden müssen (Kaluza \& Schimek 2017).

Schulentwicklungsforschung zeigt, dass „sowohl Haltungen, Werte und Grundannahmen” über Evidenzen hinaus Modelle von Qualitätssystemen und Referenzrahmen beeinflussen (Schratz et al. 2019, S. 403). Entscheidungen von Akteur*innen basieren „zunächst (nur) auf Annahmen bzw. Intuition anstatt auf Evidenz" (Schratz et al. 2019, S. 403). Eine (objektive) Identifikation von Grundproblemen und deren Ursachen stellt jedoch eine wesentliche Grundlage dar, um Schule und Unterricht zu verbessern (Schratz et al. 2019, S. 403).

Die einzelne Schule bleibt auch vor dem Hintergrund gesetzlicher Vorgaben und bestehender Qualitätsentwicklungsmodelle "Schnittpunkt, an dem die Konkrektisierung von Werten in schulischen Praktiken zu verhandeln ist" (Kaluza \& Schimek 2021, S. 115), wodurch „die Notwendigkeit der Offenlegung und Abgrenzung der normativen Setzungen, die aus unterschiedlichen Ebenen des Schulsystems zu treffen sind" (Kaluza \& Schimek 2021, S. 117f) im Rahmen von Schulentwicklungsprozessen hervorgehoben wird. Die Klärung jener Werte, die Entscheidungen und Maßnahmen zugrunde liegen, ist demnach unumgänglich, um basierend auf Evidenzen und unter Einbindung bildungswissenschaftlicher Orientierungspunkte zu Entscheidungsfindungen zu kommen (Kaluza \& Schimek 2021, S. 120). Teilschritte einer wertbasierten und evidenzinformierten diversitätsbewussten Schulentwicklung sind, in Anlehnung an Brighouse et al. (2018), ausgehend von normativen Bezugspunkten (wie z.B. der Qualitätsrahmen für Schulen in Österreich), die Anreicherung der Sprache, die Identifikation handlungsleitender Werte, die Erstellung evidenzorientierter Outcome-Prognosen sowie die Prüfung der Optionen auf ihre (exkludierenden) Wirkungen (Kaluza \& Schimek 2021, S. 119f). In diesem schulischen Aushandlungsprozess gilt es, ausgehend von subjektiven Überzeugungen und kollektiven Wertungen der einzelnen an Schule beteiligten Akteur*innen, mit Hilfe von Evidenzbasierung zu (inter-)objektiven Einstellungen zu gelangen (Zierer et al. 2019, S. 22f).

In der Zusammenschau der Ziele, Konzepte und Instrumente der Bildungsreform im Wechselspiel mit Fragen der Bildungsgerechtigkeit wird somit deutlich, dass diversitätsbewusster Schulentwicklung die systematische Verbindung von Wertediskursen und Evidenzbasierung grundzulegen ist, denn letztlich sind gerade im Kontext von Diversität „[n]icht Überzeugungen als einfache Glaubenssätze oder Wertungen als unreflektierte Tradierungen [...] Kennzeichen von Professionalität, sondern die Hinwendung zu Evidenz" (Zierer et al. 2019, S. 24). Mit der Trias von Wertediskurs, Evidenzbasierung und bildungstheoretischer Rahmung wird somit die Basis für die diversitätsbewusste Schulentwicklung (Entwicklung pädagogischer Konzepte, Antizipation von Wirkungen, ...) gelegt. 


\section{\# schuleverantworten}

Um zu beurteilen, wo Schule X steht, setzen sich die Schulleitung und die Pädagog*innen (wie im Qualitätsrahmen für Schulen vorgesehen) "gemeinsam und systematisch mit Evidenzen, Zielen, Abläufen und den Effekten von gesetzten Maßnahmen" auseinander. Dazu werden Umfragen unter Schüler*innen, Eltern und Obsorgeberechtigten, Lehrer*innen etc. gemacht und die Ergebnisse sowie bereits bestehende Daten besprochen (z.B. aus den Bildungsstandards oder künftig IKMplus). Schule X erkennt in diesem Prozess, wo der größte Handlungsbedarf ist, um einzelnen, sehr benachteiligten Schüler*innen-Gruppen besondere Unterstützung zu geben. Als erste Maßnahme haben die Schulleitung und die Pädagog*innen beschlossen, spezielle Informations- und Untersützungsangebote für Eltern bzw. Obsorgeberechtige anzubieten, um diese stärker einzubinden und mehr über die Lebenssituationen der Schüler*innen zu lernen.

Dieser Prozess und insbesondere die Auseinandersetzung mit Daten, Befragungen, Lebensrealitäten der Schüler*innen ... haben der Schulleitung und den Pädagog*innen geholfen, von individuellen teilweise sehr unterschiedlichen Haltungen zu gemeinsamen Einstellungen zu gelangen, die sie auch gut nach außen begründen können.

\section{Literaturverzeichnis}

Althoff, M. (2008). Partizipation, Steuerung und Verständigung - Schulentwicklung als dialogischer Prozess. In Althoff, M. \& Rhim, Th. (Hrsg.), Partizipation, Steuerung und Verständigung - Schulentwicklung als dialogischer Prozess (S. 121 -132), Wiesbaden: Springer VS.

Bundesministerium für Bildung, Wissenschaft und Forschung (2019). Steuerung des Schulsystems in Österreich. Weißbuch: https://www.bmbwf.gv.at/dam/jcr:07176d46-959b-4b36-a87b-8eaf142dbb24/weissbuch_steuerung_schulsystem.pdf, Stand vom 18. August 2021

Bundesministerium für Bildung, Wissenschaft und Forschung (o.J.). Qualitätsmanagementsystem in Schulen: https://www.qms.at/, Stand 19. August 2021.

Bildungsreformgesetz 2017 - Schulrecht (2017). https://www.ris.bka.gv.at/Dokument.wxe?Abfrage=Begut\&Dokumentnummer=BEGUT_COO_2026_100_2_1354973, Stand 18. August 2021

Bruneforth, M. \& Weber, Chr. \& Bacher, J. (2012). Chancengleichheit und garantiertes Bildungsminimum in Österreich. In Bundesinstitut für Bildungsforschung, Innovation \& Entwicklung des österreichischen Schulwesens (Hrsg.), Nationaler Bildungsbereich Österreich 2012. Band 2. Fokussierte Analysen bildungspolitischer Schwerpunktthemen (S. 189 - 225), Graz: Leykam.

Dahmen, B. \& Vollmer, L. (2018): Intersektionalität (über)fordert die Diversity-Praxis - zum Umgang mit dem Differenzdilemma. In Zeitschrift für Diversitätsforschung und-management, 3 (1), S. 84 - 88.

Dederich, M. (2016). 14 Bildungsgerechtigkeit. In Hedderich, I. \& Biewer, G. \& Hollenweger, J. \& Markowetz, R. (Hrsg.), Handbuch Inklusion und Sonderpädagogik (S. 92 - 96), Bad Heilbrunn: Klinkhardt. 


\section{\# schuleverantworten}

führungskultur_innovation_autonomie

Demski, D. (2017). Evidenzbasierte Schulentwicklung. Wiesbaden: Springer.

Dobusch, L. (2017). Von der Diversitäts- zur Inklusionsforschung. In Zeitschrift für Diversitätsforschung und-management, 2 (1), S. 59-63.

Giesinger, J. (2007). Was heißt Bildungsgerechtigkeit? In Zeitschrift für Pädagogik, 53 (3), S. 362-381.

Giesinger, J. (2011). Bildung als öffentliches Gut und das Problem der Gerechtigkeit. In Zeitschrift für Pädagogik, 57 (3), S. 421-437.

Kaluza, C. \& Schimek, B. (2017). Diversity Management in Schulen. Bochum und Freiburg: projektverlag.

Kaluza, C. \& Schimek, B. (2021). Inklusion aus menschenrechtlicher Perspektive im Kontext von Schulentwicklung. In Lauß, G. (Hrsg.), Ideal-Pflicht.Zumutung: Menschenrechtsbildung aus multidisziplinärer Perspektive (S. 113 - 124), Baltmannsweiler: Schneider Verlag.

OECD (2011). PISA 2009 Ergebnisse: Was macht eine Schule erfolgreich? Lernumfeld und schulische Organisation in PISA (Band IV). http://dx.doi.org/10.1787/9789264095410-de, Stand 18. August 2021

OECD (2018). Erfolgsfaktor Resilienz. http://www.oecd.org/berlin/publikationen/VSD_OECD_Erfolgsfaktor\%20Resilienz.pdf, Stand 18. August 2021.

Rawls, J. (1975). Eine Theorie der Gerechtigkeit. Frankfurt am Main: Suhrkamp.

Rolff, H.-G. (2006). Schulentwicklung, Schulprogramm und Steuergruppe. In Buchen, H. \& Rolff, H.-G. (Hrsg.), Professionswissen Schulleitung (S. 296-364), Weinheim und Basel: Beltz Verlag.

Saalfrank, W.-T. (2008). Die autonome Schule als Ort der Teilhabe. In Althoff, M. \& Rhim, Th. (Hrsg.), Partizipation, Steuerung und Verständigung - Schulentwicklung als dialogischer Prozess (S. $133-146)$, Wiesbaden: Springer VS.

Schratz, M. et al. (2019). Möglichkeiten und Grenzen evidenzorientierter Schulentwicklung. In Breit, S. et al. (Hrsg.), Nationaler Bildungsbericht Österreich 2018. Band 2. Fokussierte Analysen und Zukunftsperspektiven für das Bildungswesen: http://doi.org/10.17888/nbb2018-2, Stand 19. August 2021.

Suchań, B. \& Itzlinger-Bruneforth, U. (2017). PISA 2015. Kollaboratives Problemlösen: https://www.bifie.at/wp-content/uploads/2017/11/PISA15_Kollaboratives-Problemloesen_final.pdf, Stand 18. August 2021

Walgenbach, K. $\left(2017^{2}\right)$. Heterogenität - Intersektionalität - Diversity in der Erziehungswissenschaft. Opladen und Toronto: Verlag Barbara Budrich.

Wimmer, B. \& Altrichter, H. (2017). 13 Heterogenität als Thema von Einzelschulentwicklung. In Bohl, Th. \& Budde, J. \& Rieger-Ladich, M. (Hrsg.), Umgang mit Heterogenität in Schule und Unterricht (S. 207 - 221), Bad Heilbrunn: Verlag Julius Klinkhard

Zierer, K. \& Weckend, D. \& Schatz, C. (2019). Haltungsbildung ins Zentrum rücken. In Rotter, C. \& Schülke, C. \& Bressler, Ch. (Hrsg.), Lehrerhandeln - eine Frage der Haltung? (S. 14 - 29), Weinheim: Beltz. 


\section{Autor*innen}

Claudia Kaluza, HS-Prof. ${ }^{\text {in }}$ Mag. ${ }^{\text {a }}$ Dr. ${ }^{\text {in }}$ BEd.

Sonderschullehrerin, Hochschulprofessur für Diversitätspädagogik an der Pädagogischen Hochschule Wien, Arbeits- und Forschungsschwerpunkte: Diversität und Inklusion an Hochschulen, Inklusive Pädagogik/Sonderpädagogik, Förderschwerpunkt emotionale und soziale Entwicklung, Diversität in pädagogischen Kontexten, soziale Ungleichheit und Bildung, Diversität und Schulentwicklung.

Kontakt: claudia.kaluza@phwien.ac.at

Bernhard Schimek, HS-Prof. Mag. Dr. BEd.

Sonderschullehrer, Hochschulprofessur für Diversität und Bildungsgerechtigkeit an der Pädagogischen Hochschule Wien, Institutsleiter des Instituts für allgemeine bildungswissenschaftliche Grundlagen und reflektierte Praxis, Arbeits- und Forschungsschwerpunkte: Bildungsgerechtigkeit, Diversität und Inklusion an Hochschulen, Inklusive Pädagogik/Sonderpädagogik, Diversität in pädagogischen Kontexten, soziale Ungleichheit und Bildung, Diversität und Schulentwicklung

Kontakt: bernhard.schimek@phwien.ac.at 